\title{
On the approximation by compositions of fixed multivariate functions with univariate functions
}

\author{
by \\ Vugar E. Ismailov (Baku)
}

\begin{abstract}
The approximation in the uniform norm of a continuous function $f(\mathbf{x})=$ $f\left(x_{1}, \ldots, x_{n}\right)$ by continuous sums $g_{1}\left(h_{1}(\mathbf{x})\right)+g_{2}\left(h_{2}(\mathbf{x})\right)$, where the functions $h_{1}$ and $h_{2}$ are fixed, is considered. A Chebyshev type criterion for best approximation is established in terms of paths with respect to the functions $h_{1}$ and $h_{2}$.
\end{abstract}

1. Exposition of the problem. It is well known that in many problems of approximation of bivariate functions by sums of univariate functions the concept of a path is central. A path is a finite or infinite ordered set of points in the $x y$ plane such that the line segments joining consecutive points are of positive length and are alternately parallel to the $x$ and $y$ axes. The idea of paths, in this context, was first introduced by Diliberto and Straus [4] and exploited further in a number of works, e.g. [5, 7, 8, 10, 13]. In connection with the problem of interpolation by linear combinations of ridge functions, Braess and Pinkus [1] introduced the notion of a path with respect to distinct directions $\mathbf{a}$ and $\mathbf{b}$. This is an ordered set of points $\left(\mathbf{v}^{1}, \ldots, \mathbf{v}^{n}\right) \subset \mathbb{R}^{2}$ with edges $\mathbf{v}^{i} \mathbf{v}^{i+1}$ in alternating directions $\mathbf{a}$ and $\mathbf{b}$. These objects give a geometric method for deciding if a set of points $\left\{\mathbf{x}^{i}\right\}_{i=1}^{m}$ has the NI-property (non-interpolation property) (see [1]).

Our aim is to bring into consideration more general objects: paths with respect to two continuous functions. We will show how these objects appear in the characterization of extremal elements in the approximation problem considered below.

Let $Q$ be a compact set in $\mathbb{R}^{n}$. Consider the approximation of a function $f \in C(Q)$ by elements of the set

$$
C_{h_{1} h_{2}}=C_{h_{1} h_{2}}(Q)=\left\{g \in C(Q): g(\mathbf{x})=g_{1}\left(h_{1}(\mathbf{x})\right)+g_{2}\left(h_{2}(\mathbf{x})\right)\right\},
$$

where the functions $h_{i} \in C(Q)$ are prescribed and we vary over functions

2000 Mathematics Subject Classification: 41A30, 41A50, 41A65.

Key words and phrases: path, ridge function, best approximation.

Supported by INTAS Grant YSF-06-1000015-6283. 
$g_{i}: h_{i}(Q) \rightarrow \mathbb{R}, i=1,2$. The continuity of $g_{i}$ on $h_{i}(Q), i=1,2$, is not necessary, but the sum $g_{1}\left(h_{1}(\mathbf{x})\right)+g_{2}\left(h_{2}(\mathbf{x})\right)$ should be continuous on $Q$. It is not difficult to see that linear combinations of functions from $C_{h_{1} h_{2}}$ belong to $C_{h_{1} h_{2}}$.

Our aim is to find necessary and sufficient geometrical conditions for a function $g_{0} \in C_{h_{1} h_{2}}$ to be a best approximation to $f$, i.e. for

$$
\left\|f-g_{0}\right\|=\max _{\mathbf{x} \in Q}\left|f(\mathbf{x})-g_{0}(\mathbf{x})\right|=E\left(f, C_{h_{1} h_{2}}\right),
$$

where

$$
E(f)=E\left(f, C_{h_{1} h_{2}}\right):=\inf _{g \in C_{h_{1} h_{2}}}\|f-g\|
$$

is the error in approximating from $C_{h_{1} h_{2}}(Q)$.

In multivariate approximation theory and in some applications such as computerized tomography, statistics, and neural networks, special functions called ridge functions are widely used (see, e.g., [1-3, 9, 11, 12, 14, 16-19]). A ridge function is a multivariate function of the form $g(\mathbf{a} \cdot \mathbf{x})$, where $g$ is a univariate function, $\mathbf{a}$ is a fixed vector (direction) in $\mathbb{R}^{n}$ different from zero, $\mathbf{x} \in \mathbb{R}^{n}$ and $\mathbf{a} \cdot \mathbf{x}$ is the inner product of $\mathbf{a}$ and $\mathbf{x}$. Note that the problem of approximation by sums of two ridge functions with fixed directions is a special case of the problem considered here.

2. Main result. We begin with a definition. Let $Q$ be a compact set in $\mathbb{R}^{n}$ and $h_{i} \in C(Q), i=1,2$.

Definition 2.1. A finite or infinite set $p=\left(\mathbf{p}_{1}, \mathbf{p}_{2}, \ldots\right) \subset Q$, where $\mathbf{p}_{i} \neq \mathbf{p}_{i+1}$, with either $h_{1}\left(\mathbf{p}_{1}\right)=h_{1}\left(\mathbf{p}_{2}\right), h_{2}\left(\mathbf{p}_{2}\right)=h_{2}\left(\mathbf{p}_{3}\right), h_{1}\left(\mathbf{p}_{3}\right)=h_{1}\left(\mathbf{p}_{4}\right), \ldots$ or $h_{2}\left(\mathbf{p}_{1}\right)=h_{2}\left(\mathbf{p}_{2}\right), h_{1}\left(\mathbf{p}_{2}\right)=h_{1}\left(\mathbf{p}_{3}\right), h_{2}\left(\mathbf{p}_{3}\right)=h_{2}\left(\mathbf{p}_{4}\right), \ldots$ is called a path with respect to the functions $h_{1}$ and $h_{2}$.

In the following, we will simply say "path" instead of "path with respect to the functions $h_{1}$ and $h_{2} "$.

If in a finite path $\left(\mathbf{p}_{1}, \ldots, \mathbf{p}_{n+1}\right), \mathbf{p}_{n+1}=\mathbf{p}_{1}$ and $n$ is even, then the path $\left(\mathbf{p}_{1}, \ldots, \mathbf{p}_{n}\right)$ is said to be closed. Note that a minimal closed path may consist of two distinct points $\mathbf{p}_{1}$ and $\mathbf{p}_{2}$. In this case, the equality $h_{i}\left(\mathbf{p}_{1}\right)=h_{i}\left(\mathbf{p}_{2}\right)$ must be satisfied for both $i=1$ and $i=2$.

To each closed path $p=\left(\mathbf{p}_{1}, \ldots, \mathbf{p}_{2 n}\right)$ we associate the functional

$$
G_{p}(f)=\frac{1}{2 n} \sum_{k=1}^{2 n}(-1)^{k+1} f\left(\mathbf{p}_{k}\right) .
$$

It has the following obvious properties:

(a) If $g \in C_{h_{1} h_{2}}$, then $G_{p}(g)=0$.

(b) $\left\|G_{p}\right\| \leq 1$ and if $\mathbf{p}_{i} \neq \mathbf{p}_{j}$ for all $i \neq j, 1 \leq i, j \leq 2 n$, then $\left\|G_{p}\right\|=1$. 
We need the following auxiliary lemmas.

Lemma 2.2. If a compact set $Q$ contains closed paths, then

$$
\sup _{p \subset Q}\left|G_{p}(f)\right| \leq E\left(f, C_{h_{1} h_{2}}\right),
$$

where the sup is taken over all closed paths. Moreover, inequality (1) is sharp, i.e., there exist functions for which (1) turns into equality.

Proof. Let $p$ be any path of $Q$ and $g \in C_{h_{1} h_{2}}(Q)$. Then by the linearity of $G_{p}$ and properties (a) and (b),

$$
\left|G_{p}(f)\right|=\left|G_{p}(f-g)\right| \leq\|f-g\| .
$$

Since the left-hand and right-hand sides of (2) do not depend upon $g$ and $p$, respectively, it follows from (2) that

$$
\sup _{p \subset Q}\left|G_{p}(f)\right| \leq \inf _{g \in C_{h_{1} h_{2}}}\|f-g\| .
$$

To prove the sharpness of (1) note that if $p$ is a closed path, then there is a closed path $p^{\prime}=\left(\mathbf{p}_{1}^{\prime}, \ldots, \mathbf{p}_{2 n}^{\prime}\right)$ such that $p^{\prime} \subset p$ and all points of $p^{\prime}$ are distinct. Indeed, $p^{\prime}$ can be obtained by the following simple algorithm: if the points of $p$ are not all distinct, let $i$ and $k>0$ be the minimal indices such that $\mathbf{p}_{i}=\mathbf{p}_{i+2 k}$; delete from $p$ the subsequence $\mathbf{p}_{i+1}, \ldots, \mathbf{p}_{i+2 k}$ and call the resulting path $p$; repeat the above step until all points of $p$ are distinct; set $p^{\prime}:=p$. By Urysohn's lemma, there exists a continuous function $f^{\prime}$ such that $f^{\prime}\left(\mathbf{p}_{i}^{\prime}\right)=1$ for $i=1,3, \ldots, 2 n-1, f^{\prime}\left(\mathbf{p}_{j}^{\prime}\right)=-1$ for $j=2,4, \ldots, 2 n$, and $-1<f^{\prime}(\mathbf{x})<1$ for all $\mathbf{x} \in Q \backslash p^{\prime}$. Then

$$
G_{p^{\prime}}\left(f^{\prime}\right)=\left\|f^{\prime}\right\|=1
$$

On the other hand, it is obvious that

$$
E\left(f^{\prime}, C_{h_{1} h_{2}}\right) \leq\left\|f^{\prime}\right\| .
$$

From (3)-(5) it follows that

$$
\sup _{p \subset Q}\left|G_{p}\left(f^{\prime}\right)\right|=E\left(f^{\prime}, C_{h_{1} h_{2}}\right),
$$

and moreover sup is attained by the closed path $p^{\prime}$, so $0 \in C_{h_{1} h_{2}}$ is a best approximation to $f^{\prime}$.

For any $h \in C(Q)$, set

$$
Q_{t}=\{\mathbf{x} \in Q: h(\mathbf{x})=t\}, \quad T_{h}=\left\{t \in \mathbb{R}: Q_{t} \neq \emptyset\right\} .
$$

Lemma 2.3. Let $Q$ be a convex compact set in $\mathbb{R}^{n}$ and $f, h \in C(Q)$. Then the functions

$$
g_{1}(t)=\max _{\substack{\mathbf{x} \in Q \\ h(\mathbf{x})=t}} f(\mathbf{x}), \quad g_{2}(t)=\min _{\substack{\mathbf{x} \in Q \\ h(\mathbf{x})=t}} f(\mathbf{x}), \quad t \in T_{h},
$$

are defined and continuous on $T_{h}$. 
Proof. Observe that $T_{h}$ is a closed interval or a point. The case of a point is trivial. So, assume that $T_{h}=\left[c_{1}, c_{2}\right]$, where $c_{1} \neq c_{2}$. Suppose $g_{1}$ is not continuous on $\left[c_{1}, c_{2}\right]$ and $t_{0}$ is a point of discontinuity. First assume that $t_{0} \in$ $\left[c_{1}, c_{2}\right)$. Without loss of generality we may consider $g_{1}$ to be discontinuous from the right at $t_{0}$, i.e.

$$
\exists \varepsilon>0 \forall t^{\prime}>t_{0}, t^{\prime} \in\left[c_{1}, c_{2}\right] \exists t_{1} \in\left(t_{0}, t^{\prime}\right]:\left|g_{1}\left(t_{1}\right)-g_{1}\left(t_{0}\right)\right|>\varepsilon .
$$

Fix some $t^{\prime}$. Since $f$ is continuous on the compact set $Q$, there exist $\mathbf{y}_{0}, \mathbf{y}_{1} \in Q$ such that $g_{1}\left(t_{0}\right)=f\left(\mathbf{y}_{0}\right)$ and $g_{1}\left(t_{1}\right)=f\left(\mathbf{y}_{1}\right)$. Since $Q$ is convex, it contains the line segment $\left[\mathbf{y}_{0}, \mathbf{y}_{1}\right]$. Set

$$
Y_{0}=\left\{\mathbf{y} \in Q: f(\mathbf{y})=g_{1}\left(t_{0}\right)\right\} .
$$

It is obvious that $Y_{0}$ is closed, $\mathbf{y}_{0} \in Y_{0}$ and $\mathbf{y}_{1} \notin Y_{0}$. Write

$$
Y_{0}^{\prime}=Y_{0} \cap\left[\mathbf{y}_{0}, \mathbf{y}_{1}\right] \text {. }
$$

There is a point $\mathbf{y}_{0}^{\prime} \in Y_{0}^{\prime}$ such that

$$
\varrho\left(\mathbf{y}_{1}, Y_{0}^{\prime}\right)=\varrho\left(\mathbf{y}_{1}, \mathbf{y}_{0}^{\prime}\right) .
$$

It is clear that $h\left(\mathbf{y}_{0}^{\prime}\right)=t_{0}$ and $h\left(\mathbf{y}_{1}\right)=t_{1}$. Since $h$ is continuous on $\left[\mathbf{y}_{0}, \mathbf{y}_{1}\right]$, for any $t \in\left(t_{0}, t_{1}\right]$ there exists $\mathbf{y} \in\left(\mathbf{y}_{0}^{\prime}, \mathbf{y}_{1}\right]$ such that $h(\mathbf{y})=t$. Then it is not difficult to see that there exist sequences $\left\{t_{n}\right\} \subset\left(t_{0}, t_{1}\right]$ and $\left\{\mathbf{y}_{n}\right\} \subset\left(\mathbf{y}_{0}^{\prime}, \mathbf{y}_{1}\right]$ such that $t_{n} \downarrow t_{0}, \mathbf{y}_{n} \rightarrow \mathbf{y}_{0}^{\prime}$ and $h\left(\mathbf{y}_{n}\right)=t_{n}$. It follows from (6) that there exists a sequence $\left\{t_{n}^{\prime}\right\}$ such that $t_{0}<t_{n}^{\prime} \leq t_{n}$ and at the same time

$$
\left|g_{1}\left(t_{n}^{\prime}\right)-g_{1}\left(t_{0}\right)\right|>\varepsilon \quad \text { for all } n \text {. }
$$

For each $n$ there exist $\mathbf{y}_{n}^{\prime} \in\left(\mathbf{y}_{0}^{\prime}, \mathbf{y}_{n}\right]$ and $\mathbf{y}_{n}^{\prime \prime} \in Q$ such that $h\left(\mathbf{y}_{n}^{\prime}\right)=t_{n}^{\prime}$ and $f\left(\mathbf{y}_{n}^{\prime \prime}\right)=g_{1}\left(t_{n}^{\prime}\right)$. Then (7) can be written in the following form:

$$
\left|f\left(\mathbf{y}_{n}^{\prime \prime}\right)-f\left(\mathbf{y}_{0}^{\prime}\right)\right|>\varepsilon \quad \text { for all } n .
$$

Since $h\left(\mathbf{y}_{n}^{\prime \prime}\right)=t_{n}^{\prime}$ and $f\left(\mathbf{y}_{n}^{\prime \prime}\right)$ is the maximum of all $f(\mathbf{y})$, whereas $h(\mathbf{y})=t_{n}^{\prime}$, we find that

$$
f\left(\mathbf{y}_{n}^{\prime}\right) \leq f\left(\mathbf{y}_{n}^{\prime \prime}\right) \text { for all } n
$$

Since $\mathbf{y}_{n} \rightarrow \mathbf{y}_{0}^{\prime}, \mathbf{y}_{n}^{\prime}$ also tends to $\mathbf{y}_{0}^{\prime}$. Then $f\left(\mathbf{y}_{n}^{\prime}\right) \rightarrow f\left(\mathbf{y}_{0}^{\prime}\right)$ and $h\left(\mathbf{y}_{n}^{\prime}\right) \rightarrow$ $h\left(\mathbf{y}_{0}^{\prime}\right)$. The sequence $\left\{\mathbf{y}_{n}^{\prime \prime}\right\}$ contains a converging subsequence. Without loss of generality we may assume that $\left\{\mathbf{y}_{n}^{\prime \prime}\right\}$ itself converges to some point $\mathbf{y}^{\prime \prime} \in Q$. Then we deduce from (8) and (9) that

$$
\left|f\left(\mathbf{y}^{\prime \prime}\right)-f\left(\mathbf{y}_{0}^{\prime}\right)\right| \geq \varepsilon
$$

and

$$
f\left(\mathbf{y}_{0}^{\prime}\right) \leq f\left(\mathbf{y}^{\prime \prime}\right) .
$$

Let us prove that $f\left(\mathbf{y}^{\prime \prime}\right)=f\left(\mathbf{y}_{0}^{\prime}\right)$. Indeed, since $h\left(\mathbf{y}_{n}^{\prime \prime}\right)=t_{n}^{\prime}, \mathbf{y}_{n}^{\prime \prime} \rightarrow \mathbf{y}^{\prime \prime}$, $t_{n}^{\prime} \rightarrow t_{0}$, it follows from the continuity of $h$ that $h\left(\mathbf{y}^{\prime \prime}\right)=t_{0}$. Now, since 
$h\left(\mathbf{y}_{0}^{\prime}\right)=h\left(\mathbf{y}^{\prime \prime}\right)=t_{0}$ and $f\left(\mathbf{y}_{0}^{\prime}\right)$ is the maximum of all $f(\mathbf{y})$, whereas $h(\mathbf{y})$ $=t_{0}$, it follows from (11) that

$$
f\left(\mathbf{y}_{0}^{\prime}\right)=f\left(\mathbf{y}^{\prime \prime}\right) .
$$

The last equality together with (10) contradicts the choice of $\varepsilon$.

In the same way we can prove that $g_{1}$ is continuous at $t=c_{2}$ and $g_{2}$ is also continuous on $T_{h}$.

Definition 2.4. A finite or infinite path $\left(\mathbf{p}_{1}, \mathbf{p}_{2}, \ldots\right)$ is said to be extremal for a function $u \in C(Q)$ if $u\left(\mathbf{p}_{i}\right)=(-1)^{i}\|u\|, i=1,2, \ldots$, or $u\left(\mathbf{p}_{i}\right)=(-1)^{i+1}\|u\|, i=1,2, \ldots$

Theorem 2.5. Let $Q$ be a convex compact set in $\mathbb{R}^{n}$. A necessary and sufficient condition for a function $g_{0} \in C_{h_{1} h_{2}}$ to be a best approximation to the given function $f \in C(Q) \backslash C_{h_{1} h_{2}}$ is the existence of a closed or infinite path $l=\left(\mathbf{p}_{1}, \mathbf{p}_{2}, \ldots\right)$ extremal for the function $f_{1}=f-g_{0}$.

Proof. Necessity. Let $g_{0}(\mathbf{x})=g_{1,0}\left(h_{1}(\mathbf{x})\right)+g_{2,0}\left(h_{2}(\mathbf{x})\right) \in C_{h_{1} h_{2}}(Q)$ be a best approximation. We must show that if there is no closed path extremal for $f_{1}$, then there exists a path extremal for $f_{1}$ with infinite length (number of points). Suppose that, on the contrary, there exists a positive integer $N$ such that the length of each path extremal for $f_{1}$ is at most $N$ and no path extremal for $f_{1}$ is closed. Define

$$
f_{0}=f, \quad f_{n}=f_{n-1}-g_{1, n-1}-g_{2, n-1}, \quad n=2,3, \ldots,
$$

where

$$
\begin{aligned}
g_{1, n-1}(\mathbf{x})=g_{1, n-1}\left(h_{1}(\mathbf{x})\right)= & \frac{1}{2}\left(\max _{\substack{\mathbf{y} \in Q \\
h_{1}(\mathbf{y})=h_{1}(\mathbf{x})}} f_{n-1}(\mathbf{y})+\min _{\substack{\mathbf{y} \in Q \\
h_{1}(\mathbf{y})=h_{1}(\mathbf{x})}} f_{n-1}(\mathbf{y})\right) \\
g_{2, n-1}(\mathbf{x})=g_{2, n-1}\left(h_{2}(\mathbf{x})\right)= & \frac{1}{2}\left(\max _{\substack{\mathbf{y} \in Q \\
h_{2}(\mathbf{y})=h_{2}(\mathbf{x})}}\left(f_{n-1}(\mathbf{y})-g_{1, n-1}\left(h_{1}(\mathbf{y})\right)\right)\right. \\
& \left.+\min _{\substack{\mathbf{y} \in Q \\
h_{2}(\mathbf{y})=h_{2}(\mathbf{x})}}\left(f_{n-1}(\mathbf{y})-g_{1, n-1}\left(h_{1}(\mathbf{y})\right)\right)\right) .
\end{aligned}
$$

By Lemma 2.3, all the functions $f_{n}, n=2,3, \ldots$, are continuous on $Q$. By assumption $g_{0}$ is a best approximation to $f$. Hence $\left\|f_{1}\right\|=E(f)$. Now we show that $\left\|f_{2}\right\|=E(f)$. Indeed, for any $\mathbf{x} \in Q$,

$$
f_{1}(\mathbf{x})-g_{1,1}\left(h_{1}(\mathbf{x})\right) \leq \frac{1}{2}\left(\max _{\substack{\mathbf{y} \in Q \\ h_{1}(\mathbf{y})=h_{1}(\mathbf{x})}} f_{1}(\mathbf{y})-\min _{\substack{\mathbf{y} \in Q \\ h_{1}(\mathbf{y})=h_{1}(\mathbf{x})}} f_{1}(\mathbf{y})\right) \leq E(f)
$$

and

$$
f_{1}(\mathbf{x})-g_{1,1}\left(h_{1}(\mathbf{x})\right) \geq \frac{1}{2}\left(\min _{\substack{\mathbf{y} \in Q \\ h_{1}(\mathbf{y})=h_{1}(\mathbf{x})}} f_{1}(\mathbf{y})-\max _{\substack{\mathbf{y} \in Q \\ h_{1}(\mathbf{y})=h_{1}(\mathbf{x})}} f_{1}(\mathbf{y})\right) \geq-E(f) .
$$


In the same way, using (12) and (13), it can be shown that for any $\mathbf{x} \in Q$,

$$
-E(f) \leq f_{2}(\mathbf{x})=f_{1}(\mathbf{x})-g_{1,1}\left(h_{1}(\mathbf{x})\right)-g_{2,1}\left(h_{2}(\mathbf{x})\right) \leq E(f) .
$$

Therefore,

$$
\left\|f_{2}\right\| \leq E(f) .
$$

Since $f_{2}-f$ belongs to $C_{h_{1} h_{2}}$, we deduce from (14) that $\left\|f_{2}\right\|=E(f)$. In the same way, one can show that $\left\|f_{n}\right\|=E(f)$ for any $n$.

We now prove that if $f_{1}\left(\mathbf{p}_{0}\right)<E(f)$ for some $\mathbf{p}_{0} \in Q$, then $f_{2}\left(\mathbf{p}_{0}\right)<$ $E(f)$. We first prove that if $f_{1}\left(\mathbf{p}_{0}\right)<E(f)$, then

$$
f_{1}\left(\mathbf{p}_{0}\right)-g_{1,1}\left(h_{1}\left(\mathbf{p}_{0}\right)\right)<E(f) .
$$

Indeed, if

$$
\max _{\substack{\mathbf{y} \in Q \\ h_{1}(\mathbf{y})=h_{1}\left(\mathbf{p}_{0}\right)}} f_{1}(\mathbf{y})=E(f) \quad \text { and } \quad \min _{\substack{\mathbf{y} \in Q \\ h_{1}(\mathbf{y})=h_{1}\left(\mathbf{p}_{0}\right)}} f_{1}(\mathbf{y})=-E(f),
$$

then

$$
f_{1}\left(\mathbf{p}_{0}\right)-g_{1,1}\left(h_{1}\left(\mathbf{p}_{0}\right)\right)=f_{1}\left(\mathbf{p}_{0}\right)<E(f) .
$$

And if

$$
\max _{\substack{\mathbf{y} \in Q \\(\mathbf{y})=h_{1}\left(\mathbf{p}_{0}\right)}} f_{1}(\mathbf{y})=E(f)-\varepsilon_{1} \quad \text { and } \quad \min _{\substack{\mathbf{y} \in Q \\ h_{1}(\mathbf{y})=h_{1}\left(\mathbf{p}_{0}\right)}} f_{1}(\mathbf{y})=-E(f)+\varepsilon_{2},
$$

where $\varepsilon_{1}, \varepsilon_{2} \geq 0, \varepsilon_{1}+\varepsilon_{2} \neq 0$, then it is not difficult to verify that

$$
f_{1}\left(\mathbf{p}_{0}\right)-g_{1,1}\left(h_{1}\left(\mathbf{p}_{0}\right)\right) \leq E(f)-\frac{\varepsilon_{1}+\varepsilon_{2}}{2}<E(f) .
$$

In the same way we can prove that if $f_{1}\left(\mathbf{p}_{0}\right)-g_{1,1}\left(h_{1}\left(\mathbf{p}_{0}\right)\right)<E(f)$, then

$$
f_{1}\left(\mathbf{p}_{0}\right)-g_{1,1}\left(h_{1}\left(\mathbf{p}_{0}\right)\right)-g_{2,1}\left(h_{2}\left(\mathbf{p}_{0}\right)\right)<E(f) .
$$

So, if $f_{1}\left(\mathbf{p}_{0}\right)<E(f)$, then $f_{2}\left(\mathbf{p}_{0}\right)<E(f)$. Repeating the same techniques from (15) to (16), it can be shown that if $f_{1}\left(\mathbf{p}_{0}\right)>-E(f)$, then $f_{2}\left(\mathbf{p}_{0}\right)>$ $-E(f)$. Therefore, if $f_{2}\left(\mathbf{p}_{0}\right)=E(f)$, then $f_{1}\left(\mathbf{p}_{0}\right)=E(f)$, and if $f_{2}\left(\mathbf{p}_{0}\right)=$ $-E(f)$, then $f_{1}\left(\mathbf{p}_{0}\right)=-E(f)$. This simply means that each path extremal for $f_{2}$ will be extremal for $f_{1}$.

Now we show that if any path extremal for $f_{1}$ has length at most $N$, then any path extremal for $f_{2}$ has length at most $N-1$. Suppose that, on the contrary, there is a path extremal for $f_{2}$ of length $N$, say $q=\left(\mathbf{q}_{1}, \ldots, \mathbf{q}_{N}\right)$. We may assume that $h_{2}\left(\mathbf{q}_{N-1}\right)=h_{2}\left(\mathbf{q}_{N}\right)$. As shown above, $q$ is also extremal for $f_{1}$. Assume that $f_{1}\left(\mathbf{q}_{N}\right)=E(f)$. Then there is no $\mathbf{q}_{0} \in Q$ such that $\mathbf{q}_{0} \neq \mathbf{q}_{N}, h_{1}\left(\mathbf{q}_{0}\right)=h_{1}\left(\mathbf{q}_{N}\right)$ and $f_{1}\left(\mathbf{q}_{0}\right)=-E(f)$. Indeed, if there were such a $\mathbf{q}_{0}$ and $\mathbf{q}_{0} \notin q$, then the path $\left(\mathbf{q}_{1}, \ldots, \mathbf{q}_{N}, \mathbf{q}_{0}\right)$ would be extremal for $f_{1}$. But this would contradict our assumption that any path extremal for $f_{1}$ has length at most $N$. Also, if there were such a $\mathbf{q}_{0}$ with $\mathbf{q}_{0} \in q$, we could form some closed path extremal for $f_{1}$, contrary to our assumption. 
Hence

$$
\max _{\substack{\mathbf{y} \in Q \\ h_{1}(\mathbf{y})=h_{1}\left(\mathbf{q}_{N}\right)}} f_{1}(\mathbf{y})=E(f), \quad \min _{\substack{\mathbf{y} \in Q \\ h_{1}(\mathbf{y})=h_{1}\left(\mathbf{q}_{N}\right)}} f_{1}(\mathbf{y})>-E(f) .
$$

Therefore,

$$
\left|f_{1}\left(\mathbf{q}_{N}\right)-g_{11}\left(h_{1}\left(\mathbf{q}_{N}\right)\right)\right|<E(f) .
$$

From the last inequality it is not difficult to deduce that

$$
\left|f_{2}\left(\mathbf{q}_{N}\right)\right|<E(f) \text {. }
$$

This means that, contrary to our assumption, the path $\left(\mathbf{q}_{1}, \ldots, \mathbf{q}_{N}\right)$ cannot be extremal for $f_{2}$. Hence any path extremal for $f_{2}$ has length at most $N-1$.

In the same way, it can be shown that any path extremal for $f_{3}$ has length at most $N-2$, any path extremal for $f_{4}$ has length at most $N-3$ and so on. Finally, we conclude that there is no path extremal for $f_{N+1}$. In this case, for any $\mathbf{x} \in Q$,

$$
\left|f_{N+1}(\mathbf{x})\right|<E(f) .
$$

Since $f_{N+1}$ is continuous on $Q$, it follows from (17) that

$$
\left\|f_{N+1}\right\|<E(f) \text {. }
$$

Since the function $f_{N+1}-f$ belongs to $C_{h_{1} h_{2}}$, the last strict inequality contradicts the definition of $E(f)$. Therefore, our assumption that there does not exist an infinite path extremal for $f_{1}$ is not valid.

Sufficiency. Let $l=\left(\mathbf{p}_{1}, \ldots, \mathbf{p}_{2 n}\right)$ be a closed path extremal for $f_{1}$. It can be easily verified that

$$
\left|G_{l}(f)\right|=\left\|f-g_{0}\right\|
$$

By Lemma 2.2,

$$
\left|G_{l}(f)\right| \leq E(f) .
$$

It follows from (18), (19) and the definition of $E(f)$ that $g_{0}$ is a best approximation.

Let now $l=\left(\mathbf{p}_{1}, \mathbf{p}_{2} \ldots,\right)$ be an infinite path extremal for $f_{1}$. Without loss of generality we may assume that the points $\mathbf{p}_{i}$ are all distinct (in the other case, we could form a closed path and prove in a few lines as above that $g_{0}$ is a best approximation). Consider the sequence $l_{n}=\left(\mathbf{p}_{1}, \ldots, \mathbf{p}_{n}\right)$, $n=1,2, \ldots$, of finite paths and the path functionals

$$
F_{l_{n}}(f)=\frac{1}{n} \sum_{i=1}^{n}(-1)^{i-1} f\left(\mathbf{p}_{i}\right) .
$$

Unlike $G_{l}$, these functionals do not annihilate the set $C_{h_{1} h_{2}}$. But it can be easily verified that $\left\|F_{l_{n}}\right\|=1$ for all $n \in \mathbb{N}$. Indeed, $\left\|F_{l_{n}}(w)\right\| \leq\|w\|$ for all continuous functions $w$ over $Q$ and $\left\|F_{l_{n}}\left(w_{0}\right)\right\|=\left\|w_{0}\right\|$ for a continuous 
function $w_{0}$ taking value +1 at the points $\mathbf{p}_{i} \in l_{n}$ with $i$ odd, -1 at $\mathbf{p}_{i} \in l_{n}$ with $i$ even, and values from the interval $(-1 ; 1)$ at all other points of $Q$. By the well-known result of functional analysis (any bounded set in $E^{*}$, the dual of a separable Banach space $E$, is precompact in the weak* topology), the sequence $\left\{F_{l_{n}}\right\}_{n=1}^{\infty}$ has a weak* cluster point. Denote it by $F$. Note that for any $n \in \mathbb{N}$,

$$
\left|F_{l_{n}}\left(g_{1}+g_{2}\right)\right| \leq \frac{2}{n}\left(\left\|g_{1}\right\|+\left\|g_{2}\right\|\right)
$$

where $g_{1}(\mathbf{x})=g_{1}\left(h_{1}(\mathbf{x})\right)$ and $g_{2}(\mathbf{x})=g_{2}\left(h_{2}(\mathbf{x})\right)$. Therefore, $F(g)=0$ for all $g \in C_{h_{1} h_{2}}$. Moreover, it is clear that $\|F\| \leq 1$. From the last two properties of $F$ it follows that

$$
|F(f)|=|F(f-g)| \leq\|f-g\|
$$

for all $g \in C_{h_{1} h_{2}}$. Taking inf over $g$ on the right-hand side of (20), we obtain

$$
|F(f)| \leq E(f) .
$$

Since the paths $l_{n}$ are extremal for $f_{1}=f-g_{0}$,

$$
\left|F_{l_{n}}\left(f-g_{0}\right)\right|=\left\|f-g_{0}\right\| \text {. }
$$

Hence

$$
|F(f)|=\left|F\left(f-g_{0}\right)\right|=\left\|f-g_{0}\right\| .
$$

Now by (21) and (22), we conclude that $g_{0}$ is a best approximation.

It is well known that characterization theorems of this type are essential in approximation theory. Chebyshev was the first to prove a similar result for polynomial approximation. Khavinson [10] characterized extremal elements in a special case of the problem considered. His case allows the approximation of a continuous bivariate function $f(x, y)$ by functions of the type $\varphi(x)+\psi(y)$. It should be noted that the techniques used in the proof of Theorem 2.5 are completely different from those in [10].

REMARK. The question of existence of a best approximation from the set $C_{h_{1} h_{2}}$ to a function $f$ in $C(Q)$ (or, in other words, the proximinality of this set in the space of all continuous functions) is far from trivial. Some geometrical conditions on $Q$ sufficient for the existence of a best approximation may be found in [6]. These conditions a priori require that the mapping $h=\left(h_{1}, h_{2}\right): Q \rightarrow h_{1}(Q) \times h_{2}(Q)$ should separate points of $Q$. Necessary conditions for the proximinality of $C_{h_{1} h_{2}}$ can be easily obtained from the known general result of Marshall and O'Farrell [15] established for the sum of two algebras (see Proposition 4 in [15]). Unfortunately, there is not yet a complete answer (necessary and sufficient conditions on $Q$ ) to the above question even in the simplest case when $Q \subset \mathbb{R}^{2}$ and $h_{i}\left(x_{1}, x_{2}\right)=x_{i}$ for $i=1,2$ (see, for example, [7]). 
Acknowledgements. I would like to thank the referee, whose insightful comments helped a lot to improve the content and presentation of the paper.

\section{References}

[1] D. Braess and A. Pinkus, Interpolation by ridge functions, J. Approx. Theory 73 (1993), 218-236.

[2] M. D. Buhman and A. Pinkus, Identifying linear combinations of ridge functions, Adv. Appl. Math. 22 (1999), 103-118.

[3] E. J. Candés, Ridgelets: estimating with ridge functions, Ann. Statist. 31 (2003), 1561-1599.

[4] S. P. Diliberto and E. G. Straus, On the approximation of a function of several variables by the sum of functions of fewer variables, Pacific J. Math. 1 (1951), 195-210.

[5] N. Dyn, W. A. Light and E. W. Cheney, Interpolation by piecewise-linear radial basis functions, J. Approx. Theory 59 (1989), 202-223.

[6] A. L. Garkavi, V. A. Medvedev and S. Ya. Khavinson, On the existence of a best uniform approximation of a function of several variables by the sum of functions of fewer variables, Mat. Sb. 187 (1996), 3-14 (in Russian); English transl.: Sb. Math. 187 (1996), 623-634.

[7] - - - - On the existence of a best uniform approximation of functions of two variables by sums of the type $\varphi(x)+\psi(y)$, Sibirsk. Mat. Zh. 36 (1995), 819-827 (in Russian); English transl.: Siberian Math. J. 36 (1995), 707-713.

[8] M. v. Golitschek and W. A. Light, Approximation by solutions of the planar wave equation, SIAM J. Numer. Anal. 29 (1992), 816-830.

[9] Y. Gordon, V. Maiorov, M. Meyer and S. Reisner, On the best approximation by ridge functions in the uniform norm, Constr. Approx. 18 (2002), 61-85.

[10] S. Ja. Havinson [S. Ya. Khavinson], A Chebyshev theorem for the approximation of a function of two variables by sums of the type $\varphi(x)+\psi(y)$, Izv. Akad. Nauk SSSR Ser. Mat. 33 (1969), 650-666 (in Russian); English transl.: Math. USSR- Izv. 3 (1969), 617-632.

[11] I. G. Kazantsev, Tomographic reconstruction from arbitrary directions using ridge functions, Inverse Problems 14 (1998), 635-645.

[12] A. Kroo, On approximation by ridge functions, Constr. Approx. 13 (1997), 447-460.

[13] W. A. Light and E. W. Cheney, On the approximation of a bivariate function by the sum of univariate functions, J. Approx. Theory 29 (1980), 305-323.

[14] V. E. Maiorov, On best approximation by ridge functions, ibid. 99 (1999), 68-94.

[15] D. E. Marshall and A. G. O'Farrell, Uniform approximation by real functions, Fund. Math. 104 (1979), 203-211.

[16] K. I. Oskolkov, Ridge approximation, Chebyshev-Fourier analysis and optimal quadrature formulas, Proc. Steklov Inst. Math. 219 (1997), 265-280.

[17] P. P. Petrushev, Approximation by ridge functions and neural networks, SIAM J. Math. Anal. 30 (1998), 155-189.

[18] A. Pinkus, Approximating by ridge functions, in: Surface Fitting and Multiresolution Methods, A. Le Méhauté, C. Rabut and L. L. Schumaker (eds.), Vanderbilt Univ. Press, Nashville, 1997, 279-292. 
[19] V. Temlyakov, On approximation by ridge functions, preprint, Dept. of Math., Univ. of South Carolina, 1996, 12 pp.

Mathematics and Mechanics Institute

Azerbaijan National Academy of Sciences

Az-1141, Baku, Azerbaijan

E-mail: vugaris@mail.ru

Received May 14, 2006

Revised version August 6, 2007 